\title{
Idosos com diabetes mellitus tipo 2 e o desempenho cognitivo no teste Wisconsin de classificação de cartas (WCST)
}

\author{
Elderly people with Diabetes Mellitus Type 2 and Cognitive Performance \\ in the Wisconsin Card Sorting Test (WCST)
}

Recibido: octubre 2 de 2009 | Revisado: enero 21 de 2010 | Aceptado: marzo 14 de 2010

\author{
REgina MARIa Fernandes-LOPES ** \\ IRANI I. DE LIMA-ARGIMON **** \\ Pontificia Universidade Católica do Rio Grande do Sul,
}

Brasil

Para citar este artículo. Fernandes Lopes, R. \& De Lima Agrimon, I. (2010). Idosos com diabetes mellitus tipo 2 e o desempenho cognitivo no teste Wisconsin de classificação de cartas (WCST). Universitas Psychologica, 9 (3), 697-713.

* Artigo de pesquisa em neuropsicologia.

** Av. Assis Brasil, 3532 conj. 515/513/516, Fones: (51) 3350-5033/3350-5042/ 9967-1595/3348-3491, Jardim Lindóia- Porto Alegre-RS. E-mail: regina@ nucleomedicopsicologico.com.br

*** Av. Ipiranga, 6681-Prédio 11 - 9o andar, CEP: 90619-900-Porto Alegre-RS, Fone/Fax: (51) 3320.3500 - Ramais 3633 - 4466-4207. E-mail: argimoni@pucrs.br

\begin{abstract}
RES UMO
Estudos identificam a existência de uma conexão entre Diabetes Mellitus (DM) e demência. A hiperglicemia pode ser um fator significativo para incidência de Alzheimer e uma causa secundária de demência. Pesquisas revelaram que as funções executivas dos idosos com DM estavam mais prejudicadas do que naqueles sem DM. A Diabetes Mellitus Tipo 2 (DM2) está associada a déficits cognitivos e funcionais, e um dos instrumentos que pode ser utilizado para avaliar funções executivas é o WCST. Foi realizada uma revisão sistemática com o objetivo de verificar as características das publicações indexadas nos últimos oito anos, que abordam os temas DM2, idosos, WCST, flexibilidade cognitiva, do pensamento e funções executivas. Os resultados demonstraram que estudos envolvendo DM2, idosos e WCST são reduzidos.

Palavras-chave autor

Diabetes Tipo 2, Teste Wisconsin de Classificação de Cartas (WCST), idosos, flexibilidade cognitiva, mental e do pensamento e funções executivas.

Palabras clave descriptor

Wisconsin Card Sorting Test, Vejez, Diabetes Mellitus, Tipo 2, Enfermedad de Alzheimer.
\end{abstract}

\footnotetext{
A B S T R A C T

Studies have identified the existence of a connection between Diabetes Mellitus and dementia. High blood sugar level can be a significant factor of the incidence of Alzheimer, which could be a secondary cause of dementia. Other research revealed that the executive functions of the elderly with DM were more impaired than in those without DM. Type 2 Diabetes Mellitus (DM2) is associated with cognitive and functional deficits and, one of the tools, which can be used to assess executive functions, is the Wisconsin Card Sorting Test (WCST). A systematic review was performed with the aim of verifying the characteristics of indexed publications in the last eight years, using the following subjects: Type 2 Diabetes, elderly people, the WCST, thought and cognitive flexibility, and executive functions. Results show that studies involving DM2, elderly people and WCST are few.

Keywords author

Type 2 Diabetes, Wisconsin Card Sorting Test (WCST), elderly people, cognitive,

mental and thought flexibility and executive functions.

Keywords plus

Wisconsin Card Sorting Test, Old People Diabetes Mellitus, Type 2, Alzheimer Disease.
} 


\section{Introdução}

Estudos envolvendo idosos estão crescendo nos últimos anos, demonstrado por Argimon e Stein (2005), que referem que o envelhecimento da população é um crescente fenômeno e precisa ser mais estudado. Esse crescimento indica estar associado à melhoria das condições de vida, ao atendimento de saúde e ao avanço que a medicina tem alcançado nas últimas décadas. Presume-se que crescerá em torno de $8.95 \%$ a $18.8 \%$ o percentual de brasileiros com mais de 60 anos até o ano de 2025. Estudos da última década de Costa e Veras (2003), referem que o número de idosos acima de 60 anos de idade, no Brasil, passou de 3 milhões em 1960, para 7 milhões em 1975, e 14 milhões em 2002 (um aumento de $500 \%$ em quarenta anos); e estima-se que alcançará 32 milhões em 2020. Com o aumento da idade, torna-se comum o surgimento de dificuldades de memória associadas à doença física.

No aspecto econômico, as pessoas de Terceira Idade passam a ser vistas como improdutivas, sendo decretada, assim, sua velhice econômica e social (Amarilho \& Carlos, 2005). Passam por um processo natural de envelhecimento e, percebe-se que, no decorrer dos anos, vão sendo geradas modificações funcionais em seu organismo, favorecendo o aparecimento de doenças físicas relacionadas a esse período de vida (Nascimento, 2007). Os idosos também apresentam mais problemas de saúde, como diabetes, hipertensão arterial, problemas visuais, entre outros (Andrade, Santos \& Bueno, 2004).

Uma das doenças citadas, a Diabetes Mellitus (DM), vem crescendo em conseqüência do aumento de pessoas idosas, da ociosidade, da obesidade e do aumento de vida dos diabéticos (Freitas, Py, Cançado \& Gorzoni, 2006). A DM refere-se a uma síndrome de distúrbio metabólico de carboidratos, que envolvem a hiperglicemia, tem origem genética é subdividida em Diabetes Mellitus Tipo 1 (DM1), e a Diabetes Mellitus Tipo 2 (DM2).

A DM1 distingui-se pela destruição auto-imune celular do pâncreas, ocasionando a deficiência da insulina. É o tipo diagnosticado, com freqüência na infância e adolescência devido à destruição auto-imune das células beta do tipo 1A. No diag- nóstico, já mostra $85 \%$ das células beta destruídas, com tendência a desenvolver cetoacidose e coma, assim como um controle do metabolismo lábil. O tratamento demanda uma dose diária de insulina. A DM1 é mais comum na infância e na adolescência, ainda que possa surgir em todas as idades, firmando o diagnóstico anterior aos 30 anos de idade (Freitas et al., 2006). De todos os tipos de DM, a prevalência da DM1 é de 15\%, com tendência a cetoacidose, que é uma complicação aguda, característica do DM1, um conjugado de distúrbios metabólicos que se amplia em uma situação de deficiência insulínica grave ou absoluta, freqüentemente associada a condições estressantes que ocasionam o aumento dos hormônios contrareguladores (Foss-Freitas \& Foss, 2003).

A DM2 caracteriza-se por mais elevada prevalência em idosos e se constitui na forma mais comum, apresentando diferentes graus de deficiência e resistência à atuação da insulina. Consiste numa doença crônica que afeta idosos, sem apresentar tendência a cetoacidose. Em idosos acima de 60 anos, a prevalência oscila entre $15 \%$ a $20 \%$, com elevações nestes percentuais em idosos com mais de 75 anos. Mais de 50\% do total de pessoas com DM mostram estar acima dos 60 anos; é uma doença que está associada ao aumento de lesões macro e microvascular. Estudo de prevalência de DM no Brasil, em 1987, evidenciou uma taxa de 7,6 \%, sendo o Rio Grande do Sul o estado com maior taxa, com $12,1 \%$. A distribuição por idade foi: de 30 a 39 anos com 2,7\%; de 40 a 49 anos $-5,5 \%$; de 50 a 59 anos $-12,6 \%$; e se eleva na faixa etária de 60 a 69 anos-girando em torno de 17,4\%. Assim, mundialmente sua prevalência está crescendo, estimando-se que o número de diabéticos deverá aumentar em de 50\% até o ano de 2025 (Foss-Freitas \& Foss, 2003; Ferreira, Almeida, Siqueira \& Khawali, 2005; Lerário et al., 2008).

Com esses índices elevados da prevalência da DM, percebe-se que a DM2 é conhecida por causar prejuízos cardiovasculares, retinopatia, neuropatia periférica e enfermidades cardíacas. Nos últimos anos, houve interesse significativo em estudos sobre os efeitos da DM no cérebro (Starr \& Convit, 2007). Os pacientes com DM1, quando compa- 
rados a controles, apresentaram déficit cognitivo e diminuição do volume cerebral. Outro estudo com DM1 e cognição encontrou que a deficiência cognitiva orgânica observada se caracterizava por diminuída velocidade e flexibilidade mental (Brands, Biessels, Hann, Kapelle \& Kessels, 2005).

Diante dessas deficiências ocasionadas pela DM, Fauber (2006) mostra, em seus achados, a existência, cada vez maior da conexão entre DM e demência. O índice elevado de açúcar no sangue pode ser tanto um fator significativo para incidência de Alzheimer, como também, uma causa secundária de demência. Nos Estados Unidos, aproximadamente 18 milhões de pessoas têm DM2, e 41 milhões têm a condição conhecida como prédiabetes. DM e pressão alta, que freqüentemente estão juntas, são fatores de risco importantes e, talvez, trabalhem juntos para causar isquemia e infartos lacunares, isquemia transitória, que são uma contribuição para a demência. Num estudo longitudinal de oito anos com pessoas com DM2, os resultados de pesquisas revelam que pessoas com baixo controle da glicose tinham risco de $22 \%$ a $78 \%$ de maior probabilidade de desenvolver demência. Outras pessoas portadoras de Alzheimer e sem DM mostraram menos declínio e diminuição da velocidade do declínio.

Estudos sugerem que demência e DM2 em idosos são condições que podem estar inter- relacionadas, mas a natureza dessa associação ainda é incerta. A causa só poderá ser estabelecida através de uma pesquisa longitudinal, levando em consideração fatores de estudo sobre a cognição. Em dez estudos pesquisados (Allen, Frier \& Strachan, 2004) em que foi realizada uma combinação de avaliações de domínio cognitivo específico e um diagnóstico clínico de demência na avaliação de função cognitiva, a DM foi associada com qualquer um acelerado declínio cognitivo ou uma incidência aumentada de demência em oito de nove dos estudos baseados na população. Um destes estudos demonstrou uma relação entre DM e deterioração cognitiva vascular, mas não com outros tipos de demência. Nenhuma associação entre DM2 e declínio cognitivo foi demonstrada no caso-controle do estudo. Assim, estes estudos fornecem evidências para sustentar a tese de que as pessoas com DM2 estão com risco aumentado de deterioração cognitiva em desenvolvimento em comparação com a população geral (Allen et al., 2004).

Do mesmo modo, a DM pode afetar o sistema nervoso central resultando em deficiências cognitivas. Em pesquisa realizada por Brands et al. (2007) revelou-se impacto negativo na cognição e DM2. Este estudo envolveu extensa avaliação neuropsicológica focalizando o raciocínio abstrato, memória, atenção e funções executivas, visuoconstrução, processamento e rapidez da informação. $O$ objetivo era comparar dois grupos, um com DM1 e outro com DM2, com duração média de sete anos. Os pacientes com DM2 apresentaram significativamente mais profundas lesões e atrofia cortical em IRM. Assim, os pacientes com DM1 apresentaram um melhor desempenho ao serem pareados com pacientes com DM2 com apenas sete anos de DM.

Déficits cognitivos, incluindo eficiência psicomotora, funcionamento executivo, inteligência memória e aprendizagem, foram associados a DM2. Houve associação entre a severidade da DM2 e o grau de envolvimento no cérebro através de processamento de imagens que mostraram atrofia cortical e subcortical e aumento das lesões e prejuízo cognitivo em DM2 (Starr \& Convit, 2007). Esses estudos incluíram pacientes idosos, principalmente com controle diabético pobre.

Estudos realizados (Abel et al., 2006) com o objetivo de comparar as funções executivas de pacientes com doença degenerativa cerebelar, com pacientes com Doença de Parkinson, utilizando o instrumento Teste Wisconsin de Classificação de Cartas, revelaram que os pacientes com doença degenerativa cerebelar mostraram desempenho pior nos erros perseverativos e não perseverativos. Os resultados evidenciaram que os pacientes com doença degenerativa cerebelar apresentam um padrão de disfunção pré-frontal e déficits maiores nas funções executivas.

$\mathrm{Na}$ avaliação dos padrões de déficits cognitivos em idosos com DM, Qiu et al. (2006) estudaram 290 indivíduos idosos, sendo 40\% deles com DM. Os resultados evidenciaram que as funções cognitivas dos idosos com DM estavam mais prejudicadas 
do que naqueles sem DM. Um estudo realizado com idosos com mais de 70 anos com DM, utilizando Mini Exame do Estado Mental (MEEM) e a Escala de Depressão Geriátrica (GDS), mostrou que idosos com DM apresentam maior risco de desenvolver problemas cognitivos em relação ao grupo controle (Munshi, Grande, Hayes \& Ayres, 2006).

Para identificar precocemente declínio cognitivo em idosos, pode-se avaliar as funções executivas, que se refere às habilidades cognitivas envolvidas no planejamento, iniciação, seguimento e monitoramento de comportamentos complexos dirigidos a uma meta. Na avaliação neuropsicológica, as funções executivas implicam uma variedade de funções, como a atenção, concentração, capacidade de abstração, flexibilidade de controle mental, autocontrole, memória operacional, seletividade de estímulos (Strauss, Sherman \& Spreen, 2006).

Outra perspectiva de estudos proposta (Baudic, Dalla Barba, Thibaudet, Smagglhe Remy et al., 2006) demonstra que as funções executivas abrangem um número de habilidades cognitivas para controlar ou gerenciar o comportamento, tal como a tomada de decisão, o planejamento e monitoramento mental, e a iniciação do comportamento, a organização e a inibição. Análise realizada em pacientes com Alzheimer mostra que as funções executivas são prejudicadas precocemente já na primeira fase da doença. Além disso, a memória episódica, sustentação da atenção e déficits no funcionamento executivo precedem a deterioração.

É importante considerar que, nas ultimas décadas, tem se aprofundando o estudo sobre o papel desempenhado pelos os lobos frontais e sobre sua função, que se estende ao controle dos processos cognitivos. Essa relação infere que os lobos frontais se encarregam de uma função executiva. A função executiva, em termos gerais, é definida como processos que associam idéias simples e combinam com resolução de problemas de alta complexidade (Tiarapú-Ustrárroz \& Muñoz-Céspedes, 2005).

Lezak (2004) refere que pacientes com prejuízos na área frontal apresentam problemas de iniciativa e motivação, mostram-se incapazes de planejar metas e objetivos e não desenham planos de ação para o objetivo desejado. Assim, o funcionamento executivo ou controle executivo estão relacionados a uma série de mecanismos da otimização de processos cognitivos para resolução de situações complexas (Tiarapú-Ustrárroz \& Muñoz-Céspedes, 2005). Dessa forma, as funções executivas são responsáveis por controlar ou gerenciar o comportamento, tal como a tomada de decisão, o planejamento e monitoramento mental e a iniciação do comportamento, a organização e a inibição. Estudos mostram que em pacientes com Alzheimer, as funções executivas são prejudicadas precocemente (Baudic et al., 2006).

A flexibilidade mental faz parte das funções executivas e é aplicada à vida prática, possibilitando um monitoramento do comportamento para alternar o curso das ações de acordo com os resultados obtidos. Rocca e Lafer (2008) colocam que a flexibilidade mental é a capacidade para formar conceitos, que perseverações são compreendidas como reflexo do envolvimento, ainda que funcional, do córtex pré-frontal, porque mostra falhas na memória de trabalho e na mudança de estratégias para solução de problemas.

Para Malloy-Diniz, Sedo, Fuentes e Leite (2008, p. 198), a "flexibilidade cognitiva implica capacidade de mudar (alternar) o curso das ações ou dos pensamentos de acordo com as exigências do ambiente". Assim, a capacidade de modificar estratégias, o curso do pensamento ou dos atos de acordo com as exigências externas envolve a flexibilidade cognitiva. O Teste Wisconsin de Classificação de Cartas é um dos instrumentos mais usados para avaliação das funções executivas e, quando ocorrem muitos erros, indica dificuldades na flexibilidade cognitiva. O WCST é considerado uma significativa medida de flexibilidade cognitiva, atenção e impulsividade.

Dentro desse contexto, o teste WCST é um instrumento que avalia o raciocínio abstrato e a capacidade do individuo para gerar estratégias de solução de problemas, em resposta a condições de estimulação mutáveis. Sua criação foi em 1948, posteriormente sendo ampliado e revisado. Direcionado para a população geral passou a ser empregado, cada vez mais, como um instrumento de avaliação neuropsicológica de funções executivas que envolvem os lobos frontais (Huber, 1992). 
Entendendo a possível presença de prejuízos funcionais no desempenho da tarefa no WCST em idosos com DM2, este estudo objetiva verificar as características das publicações indexadas nos anos de 2000 a 2008, que abordam os temas Diabetes Tipo 2, idosos, flexibilidade cognitiva, funções cognitivas e Teste Wisconsin de Classificação de Cartas (WCST).

\section{Método}

Por meio de uma revisão sistemática (Coutinho, 2003), foi realizada a análise de publicações presentes nas bases de dados, a partir de um levantamento dos últimos oito anos nos indexadores e fontes Index Medline, LILACS, Psycinfo, Capes-Periódicos, Scielo, Cochrane, Proquest, Bireme, ferramentas de busca web (Yahoo, Google, Altavista), usando os descritores: Diabetes Tipo 2, Idosos, Flexibilidade Cognitiva, Flexibilidade Mental, funções executivas e Teste Wisconsin de Classificação de Cartas - WCST (Diabetes Type 2, old or older or old people or age elder or aging, Wisconsin Card Sorting Test - WCST), Wisconsin Card Sorting Test elderly, diabetes cognitive functions, diabetes type 2 elderly, WCST diabetes type 2 elderly, cognitive flexibility, cognitive flexibility aged elderly, flexibility mental elderly e Diabetes elderly. Por fim, foram avaliadas as referências dos artigos encontrados, tendo como objetivo identificar outros estudos que não tinham sido encontrados. Os estudos foram localizados, separados e analisados de acordo com o tema desta pesquisa, DM2, WCST, idosos, flexibilidade cognitiva e mental e funções executivas.

A análise do material ocorreu a partir dos seguintes critérios: base de dados, ano de publicação, país de origem, tipo de delineamento, cruzamento de variáveis, resultados e conclusões dos estudos. Os dados foram analisados de forma descritiva através de freqüências percentuais.

\section{Resultados}

Foram identificados 6756 estudos relacionados ao tema DM, sendo muitos descartados por não estarem relacionados com a temática desta pesquisa. Os estudos foram reduzidos de acordo com o tema e o ano de publicação, e apenas 30 estudos usaram o descritor "Diabetes" no seu título. Estes estudos não utilizaram no título o Teste Wisconsin de Classificação de Cartas (Wisconsin Card Sorting Test) ou WCST, sigla pela qual o teste é conhecido internacionalmente. Buscando pelos descritores Diabetes Tipo 2 e Idosos, foram encontrados 948 estudos. Cruzando os descritores Diabetes Tipo 2 e Teste Wisconsin Classificação de Cartas ou WCST foram localizados 87 estudos.

As buscas nos bancos de dados se referem aos anos de 2000 até 2008. Inicialmente, foram localizados 6756 estudos com o descritor "Diabetes elderly", sendo desta soma total 21,39\% encontrados no LILACS, 54,69\% no PubMed/Medline, 22,95\% no COCHRANE e 0,97\% no SciELO. Com as palavras-chave Diabetes type 2 elderly foram localizados apenas 3 estudos no SciELO. Assim, conforme se associaram os descritores, "WCST, Diabetes type 2, elderly", que estão relacionados mais especificamente com este trabalho, não foi encontrado nenhum estudo. Como pode ser observado na Tabela 1.

Tendo como objetivo identificar os estudos sobre Diabetes Tipo 2, idosos e WCST foram usados os descritores "Diabetes, elderly, Wisconsin Card Sorting Test" nas bases de dados, localizando-se apenas 2 estudos no período de 2000 até 2008. Os resultados podem ser observados na Tabela 1.

Trabalhos com os descritores "Wisconsin Card Sorting Test, elderly" foram encontrados 33 estudos, com 0,09\% no LILLACS, 54,54 \% no PubMed/Medline, 36,36\% no COCHRANE e nenhum estudo no SciELO. Já com as palavras- chave "Diabetes, cognitive functions" foram localizados 64 estudos, com 0,01\% no LILLACS, 32,81\% no PubMed/Medline, 62,50\% no COCHRANE e 0,03\%no SciELO. Também especificados na Tabela 1. Não foram encontrados estudos envolvendo os descritores "Cognitive flexibility", "Cognitive flexibility aged elderly" e "Flexibility mental elderly" 


\section{TABELA 1}

Freqüência simples e percentual de publicações encontradas nas bases de dados de 2000 até 2008, conforme os descritores específicos

\begin{tabular}{lcccc}
\hline \multicolumn{1}{c}{ Descritores } & LILACS & PubMed MEDLINE & COCHRANE & SciELO \\
\hline Diabetes Elderly Wisconsin Card Sorting Test & 0 & 0 & 2 & 0 \\
Wisconsin Card Sorting Test elderly & 3 & 18 & 12 & 0 \\
Diabetes cognitive functions & 1 & 21 & 40 & 2 \\
Diabetes type 2 elderly & 0 & 0 & 0 & 3 \\
WCST Diabetes type 2 elderly & 0 & 0 & 0 & 0 \\
Cognitive flexibility & 0 & 0 & 0 & 0 \\
Cognitive flexibility aged elderly & 0 & 0 & 0 & 0 \\
Flexibility mental elderly & 0 & 0 & 0 & 0 \\
Diabetes elderly & 1463 & 3712 & 1520 & 61 \\
TOTAL & 1467 & 3751 & 1574 & 66 \\
Percentual & $21,39 \%$ & $54,69 \%$ & $22,95 \%$ & $0,97 \%$ \\
\hline
\end{tabular}

Fonte: elaboração própria.

A fim de ilustrar esta revisão, foram reunidos 22 estudos na Tabela 2; destes, três estudos mais se aproximam dos objetivos do trabalho são os de: Watari et al. (2006), com amostra de 20 participantes com DM2, 34 controles, de 30 a 80 anos de idade com instrumento WCST. Os achados mostraram que diabéticos deprimidos mostram pior atenção, informação e velocidade do executivo.

Outra pesquisa realizada por Seyfaddini (2006), com uma população de 50 sujeitos com DM e 48 Controles não diabéticos, de 25 a 65 anos de idade, utilizando o WCST, MMSE, concluíram que os problemas cognitivos eram oito vezes maiores nos participantes com DM. No MMSE, não houve diferença significativa. Este estudo salienta o declínio cognitivo significativo de pacientes com DM se comparados ao grupo controle. Nas bases de dados não foram encontrados mais estudos empíricos similares a este estudo.

Finalizando, estudo envolvendo o WCST realizado por Zhao e Liu (2000), em 30 pacientes com DM2 e 30 controles, de 40 a 60 anos, relata que os pacientes com DM2 mostraram diminuída memória, atenção, habilidade de generalizar e flexibilidade do pensamento e, ainda, inibição causada por hipofunção de fluxo sangüíneo cerebral frontal e temporal.

$\mathrm{Na}$ Tabela 3, foram reunidos estudos de revisão considerando os indivíduos com DM2 associada a alterações quanto ao declínio cognitivo, risco de demência e risco a depressão. Estes estudos também referem funções de memória verbal, aprendizagem, eficiência psicomotora.

Buscando artigos publicados no idioma português, foram localizados 5 estudos desde o ano de 2002. Hamdan e Bueno (2005) compararam e relacionaram o desempenho de idosos com comprometimento Cognitivo Leve e Demência do Tipo Alzheimer em testes de funções executivas e de memória episódica verbal. Foram utilizados os testes para avaliação das funções executivas: Wisconsin Card Sorting Test, Random Number Generation, Trail Making Test, Reading Span Test, Brow-Peterson Test e Fluência Verbal semântica e fonológica. Treze idosos do grupo controle foram equiparados quanto à idade e escolaridade com 


\section{TABELA 2}

Resultados das publicações encontradas dos estudos empíricos sobre DM2 associados a alterações cognitivas, no período de 2000 a 2008

\begin{tabular}{|c|c|c|c|}
\hline Autores/Ano/ & Amostra & $\begin{array}{l}\text { Instrumentos de } \\
\text { Avaliação }\end{array}$ & Resultados \\
\hline Origem & $\begin{array}{l}98 \text { com DM2 } \\
47 \text { controles }\end{array}$ & $\begin{array}{l}\text { DNART: QI pré-mór- } \\
\text { bido }\end{array}$ & $\begin{array}{l}\text { Comparação entre perfusão, DM e função cognitiva. Re- } \\
\text { dução total perfusão cerebral funcionamento cognitivo } \\
\text { prejudicado em DM2. }\end{array}$ \\
\hline $\begin{array}{l}\text { Brands et al., } \\
2007 \text { (Ne- } \\
\text { therlands) }\end{array}$ & $\begin{array}{l}40 \mathrm{DM} 1 \\
40 \mathrm{DM} 2\end{array}$ & $\begin{array}{l}\text { Raven, WAIS-III, } \\
\text { B.Corsi, Stroop, RAV- } \\
\text { LT, LLT, ROCFT, TMT- } \\
\text { A e B, BSAT, FV }\end{array}$ & $\begin{array}{l}\text { Pacientes com DM2 apresentaram significativamente } \\
\text { lesões mais profundas e atrofia cortical em MRIs. Assim, } \\
\text { os pacientes com DM1 apresentaram um melhor desem- } \\
\text { penho ao serem pareados com pacientes com DM2 com } \\
\text { apenas sete anos de DM. }\end{array}$ \\
\hline $\begin{array}{l}\text { Bruehl et al., } \\
2007 \text { (Ale- } \\
\text { manha) }\end{array}$ & $\begin{array}{l}30 \text { com DM2, } \\
30 \text { controles } \\
43 \text { a } 74 \text { anos }\end{array}$ & $\begin{array}{l}\text { CVLT, DS do WMS-R, } \\
\text { CWAT, HAM-D Stro- } \\
\text { op, DSS, WAIS-R }\end{array}$ & $\begin{array}{l}\text { A DM2 mostrou déficits na memória declarativa. O hipo- } \\
\text { campo é prejudicado. }\end{array}$ \\
\hline $\begin{array}{l}\text { Gold et al., } \\
2007 \text { (USA) }\end{array}$ & $\begin{array}{l}23 \mathrm{DM} 2 \\
23 \text { controles } \\
45 \text { a } 70 \text { anos }\end{array}$ & $\begin{array}{l}\text { CVLT, DS, WMS-R, } \\
\text { IDPR, GMT, PST, SILS }\end{array}$ & $\begin{array}{l}\text { DM2 associado à redução precoce do hipocampo. DM1 } \\
\text { e DM2 interferem na cognição e cérebro. Aumentada } \\
\text { atrofia e déficits cerebrais, de velocidade de processamen- } \\
\text { to, inteligência e atenção. DM2 mostra a deterioração } \\
\text { de memória e a velocidade de processo verbal, atenção e } \\
\text { velocidade psicomotora. }\end{array}$ \\
\hline $\begin{array}{l}\text { Harten et al., } \\
2007 \text { (Holan- } \\
\text { da) }\end{array}$ & $\begin{array}{l}45 \text { DM2 HAS } \\
45 \text { DM2 } \\
44 \text { controles } \\
\text { média: } 73 \text { anos }\end{array}$ & $\begin{array}{l}\text { MRI, WML (neuro- } \\
\text { imagem) }\end{array}$ & $\begin{array}{l}\text { Tendência para pontuações de atrofia mais altas em DM2 } \\
\text { em relação aos controles. DM2 é um fator de risco inde- } \\
\text { pendente na idade avançada. }\end{array}$ \\
\hline $\begin{array}{l}\text { Harten et al., } \\
2007 \text { (Holan- } \\
\text { da) }\end{array}$ & $\begin{array}{l}92 \text { DM2 } \\
44 \text { controles, } \\
\text { média: } 73 \text { anos }\end{array}$ & $\begin{array}{l}\text { MRI, WML (neuro- } \\
\text { imagem) }\end{array}$ & $\begin{array}{l}\text { Duração da DM2 significativamente associada à deficiên- } \\
\text { cia orgânica cognitiva, diminuída função cognitiva em } \\
\text { domínios cognitivos diferentes, memória menos afetada. }\end{array}$ \\
\hline $\begin{array}{l}\text { Verdelho } \\
\text { et al., } 2007 \\
\text { (Portugal) }\end{array}$ & $\begin{array}{l}638 \text { DM } \\
\text { Média: } 74 \text { anos }\end{array}$ & $\begin{array}{l}\text { MMSE, VADAS-Cog, } \\
\text { Stroop, TMT }\end{array}$ & $\begin{array}{l}\text { Funcionamento executivo pior, atenção, velocidade e con- } \\
\text { trole motor. DM interfere no funcionamento executivo. } \\
\text { Necessidade de controlar fatores de risco a fim de prevenir } \\
\text { o declínio cognitivo em idosos. }\end{array}$ \\
\hline $\begin{array}{l}\text { Arvanitakis } \\
\text { et al., } 2006 \\
\text { (USA) }\end{array}$ & $\begin{array}{l}127 \text { DM } \\
824 \text { controles } \\
>55 \text { anos }\end{array}$ & $\begin{array}{l}\text { WLM, WLR, WLR, } \\
\text { CERAD, M: WMS, } \\
\text { EBS, VF, VT, JLO, } \\
\text { NART, SPM, DS }\end{array}$ & $\begin{array}{l}\text { Estudos sugerem que a DM está associada a declínio nas } \\
\text { funções cognitivas em idosos. }\end{array}$ \\
\hline $\begin{array}{l}\text { Arvanitakis } \\
\text { et al., } 2006 \\
\text { (USA) }\end{array}$ & $\begin{array}{l}116 \text { com DM2, } \\
766 \text { controles } \\
\text { idosos }\end{array}$ & $\begin{array}{l}\text { CERAD, Raven, M. } \\
\text { Lógica } 1 \text { e } 2 \text { (WMS), } \\
\text { EBS, NC BNT, BNT, } \\
\text { RT, FV, Dígitos, PS, } \\
\text { Stroop, SMDT }\end{array}$ & $\begin{array}{l}\text { Resultados sugerem que DM2 está associada com deterio- } \\
\text { ração cognitiva, especialmente em memória semântica e } \\
\text { velocidade perceptiva. }\end{array}$ \\
\hline
\end{tabular}




\begin{tabular}{|c|c|c|c|}
\hline Autores/Ano/ & Amostra & $\begin{array}{l}\text { Instrumentos de } \\
\text { Avaliação }\end{array}$ & Resultados \\
\hline $\begin{array}{l}\text { Munshi et al., } \\
2006 \text { (USA) }\end{array}$ & $\begin{array}{l}60 \text { com DM2, } \\
70 \text { a } 93 \text { anos }\end{array}$ & $\begin{array}{l}\text { MEEM, CDT, CIB, } \\
\text { GDS }\end{array}$ & $\begin{array}{l}\text { Déficits nas áreas de eficiência psicomotora, cognição } \\
\text { global, memória episódica, semântica e de trabalho. } \\
\text { Anormalidades em funções cognitivas mediadas por lobo } \\
\text { frontal (funções executivas), comportamentos complexos: } \\
\text { resolução de problemas, planejamento, organização, pers- } \\
\text { picácia, raciocínio e atenção. }\end{array}$ \\
\hline $\begin{array}{l}\text { Suzuki et al., } \\
2006 \text { (Japão) }\end{array}$ & $\begin{array}{l}13 \text { DM2 } \\
65 \text { a } 77 \text { anos }\end{array}$ & $\begin{array}{l}\text { MMSE, Word List, } \\
\text { ADAS, DST: WAIS-R, } \\
\text { Stroop }\end{array}$ & $\begin{array}{l}\text { Idosos com DM2 com glicemia elevada mostraram escores } \\
\text { menores no MMSE. }\end{array}$ \\
\hline $\begin{array}{l}\text { Seyfaddini, } \\
2006 \text { (Irã) }\end{array}$ & $\begin{array}{l}50 \text { DM e } 48 \\
\text { Controles, } \\
25 \text { a } 65 \text { anos }\end{array}$ & WCST, MMSE & $\begin{array}{l}\text { Problemas cognitivos eram oito vezes maiores nos pacien- } \\
\text { tes com DM. No MMSE não houve diferença significativa. } \\
\text { Estudo confirma o declínio cognitivo. }\end{array}$ \\
\hline $\begin{array}{l}\text { Watari et al., } \\
2006 \text { (USA) }\end{array}$ & $\begin{array}{l}20 \text { com DM2 } \\
34 \text { controles } \\
30 \text { a } 80 \text { anos }\end{array}$ & $\begin{array}{l}\text { WCST, MMSE, Stro- } \\
\text { op, RMT, TMT-A/B, D, } \\
\text { S, CB, SNL, PS, RM, } \\
\text { CVLT, Rey-O, HAM- } \\
\text { D, FAZ RMT, CVFT }\end{array}$ & $\begin{array}{l}\text { Diabéticos deprimidos mostram pior atenção, informação } \\
\text { e velocidade cognitiva. }\end{array}$ \\
\hline $\begin{array}{l}\text { Kuo et al., } \\
2005 \\
\text { ( EUA) }\end{array}$ & $\begin{array}{l}2.802 \\
65 \text { a } 94 \text { anos }\end{array}$ & DSST & $\begin{array}{l}\text { A hipertensão e DM são associados com acelerado declí- } \\
\text { nio executivo. Precisa determinar se modificação de risco } \\
\text { cardiovascular melhora declínio cognitivo e funcional nas } \\
\text { pessoas de idade avançada. }\end{array}$ \\
\hline $\begin{array}{l}\text { Hassing et al., } \\
2004 \text { (USA) }\end{array}$ & $\begin{array}{l}36 \text { com DM2 } \\
238 \text { controles } \\
80 \text { a } 93 \text { anos }\end{array}$ & $\begin{array}{l}\text { MEEM, DSS, PMT, } \\
\text { Dígitos, Cubos do } \\
\text { WAIS-R, P. Speed, MR } \\
\text { IT, VM, M. Lógica da } \\
\text { WMS }\end{array}$ & $\begin{array}{l}\text { A conclusão que DM2 é associada com acelerado declínio } \\
\text { cognitivo em idosos que resultar em demência. DM2 como } \\
\text { associado com risco aumentado de declínio cognitivo em } \\
\text { idoso. }\end{array}$ \\
\hline $\begin{array}{l}\text { Kanaya et al., } \\
2004 \text { (USA) }\end{array}$ & $\begin{array}{l}999 \text { sujeitos de } \\
42 \text { a } 89 \text { anos }\end{array}$ & MEEM, FV, TMT-B & $\begin{array}{l}\text { Mulheres com DM mostram acelerado declínio cognitivo } \\
\text { e na memória semântica, observado através de um estudo } \\
\text { de coorte. Controle da glicose previne e poderia melhorar } \\
\text { o declínio. }\end{array}$ \\
\hline $\begin{array}{l}\text { Bruce et al., } \\
2001 \\
\text { (Australia) }\end{array}$ & $\begin{array}{l}63 \text { com DM2 } \\
>\text { de } 70 \text { anos }\end{array}$ & MEEM, IQCODE & $\begin{array}{l}\text { Taxa alta de demência nas pessoas idosas com DM pode } \\
\text { ser explicada pela alta de hipertensão nesta amostra. }\end{array}$ \\
\hline $\begin{array}{l}\text { Mogi et al., } \\
2003 \text { (Japão) }\end{array}$ & $\begin{array}{l}69 \mathrm{DM} 2 \\
27 \text { Controles } \\
60 \text { a } 85 \text { anos }\end{array}$ & $\begin{array}{l}\text { MMSE, WLR, DST, } \\
\text { Stroop, WTH }\end{array}$ & $\begin{array}{l}\text { Idosos japoneses com DM2, especialmente os que fazem } \\
\text { uso de insulina, apresentaram função cognitiva pobre. }\end{array}$ \\
\hline $\begin{array}{l}\text { Cosway et al., } \\
2001 \text { ( Euro- } \\
\text { pa) }\end{array}$ & $\begin{array}{l}38 \text { DM2 } \\
38 \text { controles } \\
40 \text { a } 75 \text { anos }\end{array}$ & $\begin{array}{l}\text { NART, RAVEN, } \\
\text { WMS-R, AVLT, BVF, } \\
\text { I.T., VCD, }\end{array}$ & $\begin{array}{l}\text { Duração DM2 correlaciona-se significativamente a me- } \\
\text { didas mais baixas de memória. Hipertensão e depressão } \\
\text { podem contribuir precocemente para o declínio cognitivo. }\end{array}$ \\
\hline $\begin{array}{l}\text { Grods- } \\
\text { tein et al., } \\
2001 \text { (USA) }\end{array}$ & $\begin{array}{l}82 \text { com DM2, } \\
2.374 \text { total } \\
70 \text { a } 78 \text { anos }\end{array}$ & $\begin{array}{l}\text { MEEM, TICS, EBMT, } \\
\text { FV }\end{array}$ & $\begin{array}{l}\text { Mulheres com DM2 pontuaram menos nos testes cogni- } \\
\text { tivos do que as sem DM. DM2 relacionada a pontuações } \\
\text { mais baixas em vários aspectos da função cognitiva. A } \\
\text { duração da diabete pode ser associada com pontuações } \\
\text { mais pobres. }\end{array}$ \\
\hline
\end{tabular}




\begin{tabular}{|c|c|c|c|}
\hline Autores/Ano/ & Amostra & $\begin{array}{l}\text { Instrumentos de } \\
\text { Avaliação }\end{array}$ & Resultados \\
\hline $\begin{array}{l}\text { Sinclair et al., } \\
2000 \text { (Aus- } \\
\text { trália) }\end{array}$ & $\begin{array}{l}396 \text { DM, } 393 \\
\text { Controles } \\
>65 \text { anos }\end{array}$ & $\begin{array}{l}\text { MMSE,Clock Drawing } \\
\text { Test }\end{array}$ & $\begin{array}{l}\text { Idosos com DM2 mostram significativa deficiência orgâni- } \\
\text { ca cognitiva associada com habilidade mais pobre da pró- } \\
\text { pria doença e maior dependência. Avaliação de rotina na } \\
\text { cognição em idosos com DM é recomendada. }\end{array}$ \\
\hline $\begin{array}{l}\text { Zhao, } 2000 \\
\text { (China) }\end{array}$ & $\begin{array}{l}30 \text { DM2, } \\
30 \text { Controles } \\
40 \text { a } 60 \text { anos }\end{array}$ & $\begin{array}{l}\text { WCST, WAIS-R, } \\
\text { WMS-R, TMT-A }\end{array}$ & $\begin{array}{l}\text { Diminuída memória, atenção, habilidade de generalizar e } \\
\text { flexibilidade do pensamento. Inibição causada por hipo- } \\
\text { função de fluxo sangüíneo cerebral frontal e temporal. }\end{array}$ \\
\hline
\end{tabular}

Fonte: elaboração própria.

Notas = ADAS:Alzheimer's Disease Assessment Scale; ADL: Instrumental Activities of Daily Living; ADL: Activities of Daily Living; AVLT: Rey Auditory Verbal Learning Test; BDAE: Boston Diagnostic Aphasia Examination; BNT: Boston Naming Test; BVFT: Borkowski; BVRT: Benton Visual Retention Test; CERAD: Consortium to Establish a Registry for Alzheimer's Disease; BSAT: Brixton Spatial Anticipation Test; CDT: Clock-Draving Test; CIB: Clock-in-a-box; CNTAB: Cambridge Neuropsychological Test Automated Battery; CVAT:Controlled Words Association Test; CVLT: Califórnia Verbal Learning Test; CRT: Choice Reactikon Time; DM: Diabetes Mellitus; DM1: Diabetes Mellitus Tipo 1; DM2: Diabetes Mellitus Tipo 2; DNART (QI): Dutch National Adult Reading Test ; DS: Digit Span da WMS; DR: Desenho do Relógio; DSST ou DSS:Digit Symbol Substituition Test ( WAIS-R); DST: Digit Symbol Test; DAS: Disease Assessment Scale; EBS: East Boston Story; EBMT: East Boston Memory Test; ERPs: Event Related Potentials; FSR: Framingham Stroke Risk; FV: Fluência Verbal; GDS: Escala de Depressão Geriátrica; GMT: Guild Memory Test; HAM-D: Hamilton Depression Scale; IQCODE: Informant Questionnaire for Cognitive Decline in the Elderly; IT: Information Task; I.T.: Inspection Time; JLO: Judgment of Line Orientation; LLT: Location Learning Test; LSOA II: Second Longitudinal Study; MDRS: Mattis Dementia Rating Scale; MV: Memória Verbal; MEEM:Mini Exame do Estado Mental; MR: Memory-in-Reality; NART: National Adult Reading Test; NC: Number Comparison; PST ou P. Speed: Perceptual Speed Test; PMT: Picture Memory Test; PS: Procurar Símbolos -WAIS-III; RDT: Rosen drawing Test; Rey-O: ReyOsterrrieth Complex Figure; RT:Reading Test; RVAT: Rey Verbal Auditory Test; SRT: Selective Reminding Test; SILS: Shipley Institute of Living Scale; SDMT: Symbol Digit Modalities Test; SPM: Standard Progressive Matrices; TAAVR: Teste de Aprendizado Auditivo Verbal de Rey; TICS: Telephone Interview for Cognitive Status; TMT- B: Trail Making Test Parte B; TMT-A: Trail Making Test Parte A; VCD: Visual Change Detection; VFT: Verbal Fluency Test; VLT: Verbal Learning Test; VMT:Verbal Meaning Test; VT: Vocabulary test; WAIS-R: Escala de Inteligência Wechsler-Revisada; WCST: Wisconsin Card Sorting Test; WMS-R:Escala Wechsler de Memória Revisada; WLM: Word List Memory; WLR: Word List Recall; WLR: Word List Regnition.

\section{TABELA 3}

Resultados dos estudos de revisão de literatura sobre DM2 e alterações cognitivas relacionadas aos anos de 2000 até 2008

\begin{tabular}{|c|c|c|c|}
\hline Autor(s) & Ano & Origem estudo & Resultados \\
\hline Biessels et al. & 2008 & USA & $\begin{array}{l}\text { A natureza e severidade de DM2 relacionada com mudanças na } \\
\text { função cognitiva foram esclarecidas. Risco de declínio cognitivo ace- } \\
\text { lerado e demência atribuída a DM. }\end{array}$ \\
\hline Kodl e Seaquist & 2008 & EUA & $\begin{array}{l}\text { A deficiência orgânica pode ser listada como uma das muitas com- } \\
\text { plicações da DM, junto com retinopatia, neuropatia, nefropatia, e } \\
\text { doença cardiovascular. A deficiência orgânica cognitiva é só parcial- } \\
\text { mente compreendida. }\end{array}$ \\
\hline Aszalós & 2007 & Hungria & $\begin{array}{l}\text { DM2 risco aumentado de Doença de Alzheimer. Prevalência de } \\
\text { depressão é mais alta em pacientes com DM e depressão é um fator } \\
\text { de risco para DM. Presença simultânea de DM e depressão tendem a } \\
\text { piorar o curso de ambos. }\end{array}$ \\
\hline
\end{tabular}




\begin{tabular}{|c|c|c|c|}
\hline Autor(s) & Ano & Origem estudo & Resultados \\
\hline Biessels et al. & 2007 & Holanda & $\begin{array}{l}\text { DM pode levar a complicações em longo prazo no cérebro. Alte- } \\
\text { rações no funcionamento cognitivo. Deteriorações da cognição } \\
\text { acontecem acima de } 65 \text { anos. }\end{array}$ \\
\hline Starr e Convit & 2007 & USA & $\begin{array}{l}\text { Efeitos da DM2 no cérebro estão tornando um tópico de grande inte- } \\
\text { resse. Resistência de insulina e de DM2 são associadas com deterio- } \\
\text { rações na cognição e anormalidades em neuroimagem, independente } \\
\text { de comorbidades como hipertensão. }\end{array}$ \\
\hline Sakurai e Yokono & 2006 & Japão & $\begin{array}{l}\text { Atrofia subcortical nos lobos parietais e tálamo, que constituem pre- } \\
\text { ditores de deficiência cognitiva orgânica na DM em idade avançada. } \\
\text { Deficiência cognitiva nos aspectos de atenção, memória, velocidade } \\
\text { de informações e memória de trabalho. }\end{array}$ \\
\hline Fauber & 2006 & USA & $\begin{array}{l}\text { Piora e acelera Doença de Alzheimer, maior probabilidade desenvol- } \\
\text { ver demência. }\end{array}$ \\
\hline Kouta & 2006 & Japão & $\begin{array}{l}\text { Declínio cognitivo associado a DM como uma importante comorbi- } \\
\text { dade. Controle da hiperglicemia para manter cérebro saudável. }\end{array}$ \\
\hline Van den Berg et al. & 2006 & USA & $\begin{array}{l}\text { Associação entre DM2 e deficiência cognitiva e demência em DM2 } \\
\text { foram tratados estatisticamente. DM associado ao risco de demência. }\end{array}$ \\
\hline Bauduceau et al. & 2005 & França & $\begin{array}{l}\text { DM contribui para declínio cognitivo, e quanto maior, mais alta a } \\
\text { gravidade maior de eventos cerebrais, de demência e de AVC. }\end{array}$ \\
\hline Messier & 2005 & USA & $\begin{array}{l}\text { DM associada a prejuízos nos processos cognitivos em idosos apres- } \\
\text { sam a progressão para demência. Os déficits cognitivos mais comuns } \\
\text { são diminuições em processo velocidade e memória verbal, que pode } \\
\text { se estender para outros aspectos da cognição com o avanço da idade. } \\
\text { Depois de } 70 \text { anos, DM parece produzir declínio cognitivo mais rápi- } \\
\text { do e pode aumentar a ocorrência de mudanças patológicas associadas } \\
\text { com doença da demência vascular ou Alzheimer. }\end{array}$ \\
\hline Allen & 2004 & Europa & $\begin{array}{l}\text { E estudos longitudinais sustentam a visão de que as pessoas com DM } \\
\text { estão em risco aumentado de desenvolver deterioração cognitiva } \\
\text { comparado à população geral. Isto pode ser relacionado à duração da } \\
\text { DM. }\end{array}$ \\
\hline Awad et al. & 2004 & USA & $\begin{array}{l}\text { DM2 que mantém controle, tem impacto menor nas funções cogniti- } \\
\text { vas antes da idade de } 70 \text { anos. Pobre controle e a presença de doença } \\
\text { micro e macrovascular pode interagir produzir primeiros déficits } \\
\text { cognitivos em idosos (acima de } 70 \text { anos). DM provavelmente intera- } \\
\text { ge com outros processos da doença vascular e Alzheimer e acelerar o } \\
\text { declínio cognitivo. }\end{array}$ \\
\hline Wild & 2004 & Austrália & $\begin{array}{l}\text { A prevalência de DM para todas as idades-grupo mundiais estimados } \\
\text { era de } 2.8 \% \text { em } 2000 \text { e } 4.4 \% \text { em } 2030 \text {. O número total das pessoas } \\
\text { com DM é projetado para } 171 \text { milhões em } 2000 \text { a } 366 \text { milhões em } \\
\text { 2030. A prevalência de DM é mais alta em homens do que em mul- } \\
\text { heres, mas existem mais mulheres com DM que homens. Os países } \\
\text { em desenvolvimento apresentam uma expectativa de dobrar entre } \\
2000 \text { e 2030. Indicam que a "epidemia de diabete" continuará en- } \\
\text { quanto que os níveis de obesidade permanecerem. }\end{array}$ \\
\hline
\end{tabular}




\begin{tabular}{lcll}
\hline \multicolumn{1}{c}{ Autor(s) } & Ano & Origem estudo & \multicolumn{1}{c}{ Resultados } \\
\hline $\begin{array}{l}\text { Sastre e Evans } \\
2003\end{array}$ & USA & $\begin{array}{l}\text { Declínio cognitivo mais cedo que as pessoas saudáveis. Testes futuros } \\
\text { deveriam incluir medidas de função cognitiva como resultado. }\end{array}$ \\
$\begin{array}{l}\text { Asimakopoulou e } \\
\text { Hampson }\end{array}$ & 2002 & Inglaterra & $\begin{array}{l}\text { DM2 associada com declínio cognitivo. Declínio nos aspectos da } \\
\text { função cognitiva, como: memória verbal, aprendizagem, eficiência } \\
\text { psicomotora e funcionamento cognitivo. }\end{array}$ \\
$\begin{array}{l}\text { Biessels et al. } \\
2002\end{array}$ & Europa & $\begin{array}{l}\text { DM como fator de risco para declínio cognitivo e demência em ido- } \\
\text { so. Desafio das próximas décadas será para desvendar a interação } \\
\text { complexa entre os mecanismos de envelhecer e DM. }\end{array}$ \\
\hline
\end{tabular}

Fonte: elaboração própria.

nove idosos com comprometimento Cognitivo Leve e oito idosos com Demência Tipo Alzheimer. Nos resultados, foram encontradas diferenças e associações estatisticamente significativas em relação aos testes de funções executivas entre os grupos pesquisados. No Wisconsin Card Sorting Test, $o$ número de categorias completadas pelos idosos controles foi significativamente maior em relação aos idosos com Demência do Tipo Alzheimer, mas não em relação aos idosos com Comprometimento Cognitivo Leve.

A segunda publicação nacional identificada nestes últimos cinco anos teve como objetivo a associação entre dependência de cocaína e crack e desempenho cognitivo, de Cunha, Nicastri, Gomes, Moino e Peluso (2004). Uns dos instrumentos utilizados foram: Wisconsin Card Sorting Test - WCST, Vocabulário - Dígitos Diretos - DD e Dígitos Indiretos - DI WAIS-R, Trail Making Test TMT, Stroop Color Word Test - SCWT e Buschke Selective Reminding Test-BSRT. Estes instrumentos foram aplicados em 15 dependentes de cocaína, em abstinência por duas semanas, em tratamento em regime de internação, e em 15 sujeitos controles, não usuários de drogas, pareados por idade, sexo, escolaridade, nível socioeconômico, lateralidade e QI. Achados preliminares mostraram significação estatística $(p<0,05)$ em testes capacidade de aprendizagem, funções executivas, de atenção, fluência verbal, memória visual e memória verbal. O estudo mostrou alterações no desempenho dos pacientes dependentes de cocaína/crack no WCST. Os resultados indicaram que o abuso de cocaína está associado a déficits cognitivos, semelhantes aos que ocorrem em transtornos cognitivos, possivelmente relacionados a problemas em áreas cerebrais temporais e pré-frontais.

O terceiro artigo nacional utilizando o WCST contou com uma amostra de 32 indivíduos com tentativa de suicídio, pareados a 32 indivíduos sem história de tentativa de suicídio. Keller e Werlang (2005) buscaram avaliar a flexibilidade para resolução de problemas em indivíduos que tentaram suicídio. Os instrumentos utilizados foram: Teste Wisconsin de Classificação de Cartas (WCST), MINI- Mini-International Neuropsychiatric Interview, Teste de Stroop de Cores e Palavras, BHSEscala de Desesperança de Beck, subtestes Vocabulário, Cubos e Códigos (WAIS-III). No Teste Stroop e nas categorias do WCST dos sujeitos com tentativa de suicídio, os escores foram inferiores. Os achados mostraram que tentadores de suicídio apresentam maiores níveis de desesperança, menos flexibilidade para resolução de problemas do que os indivíduos sem história de tentativa de suicídio; o que demonstra uma associação entre a desesperança e a deficiência na flexibilidade para resolução de problemas.

O quarto artigo que utilizou o teste Wisconsin de Classificação de cartas foi de Abel et al. (2006), buscou comparar as funções executivas de pacientes com Doença de Parkinson e com pacientes com doença degenerativa cerebelar. Os resultados indicaram que os pacientes com doença degenerativa cerebelar evidenciaram desempenho pior nos erros não perseverativos e perseverativos. Os 
achados mostraram que os pacientes com doença degenerativa cerebelar apresentam déficits maiores nas funções executivas e disfunção pré-frontal.

O quinto artigo publicado no Brasil utilizando o WCST ( Rigoni, Oliveira, Moraes \& Zambom, 2007), teve como objetivo comparar o desempenho das funções cognitivas de 30 usuários de maconha e 30 não usuários de 14 a 17 anos de idade. Foi utilizado também, entrevista estruturada, o Screening Cognitivo (WISC-III e WAIS-III) e as Figuras Complexas de Rey. Os resultados mostraram que houve diferença estatisticamente significativa no desempenho dos dois grupos em todos os instrumentos aplicados, com maior prejuízo no desempenho do WCST e do subteste Códigos ( WAIS-III e WISC-III).

$\mathrm{Na}$ literatura internacional, o levantamento sobre estudos utilizaram o Teste Wisconsin de Classificação de Cartas (WCST) mostrou que os idosos com DM tendem a apresentar prejuízos significativos, sustentando a hipótese da existência da relação entre DM e deficiência orgânica (Seyfaddini, 2006). As deteriorações cognitivas caracterizam-se, especialmente, por diminuída velocidade mental e flexibilidade mental. O estudo de coorte foi realizado para distinguir a relação da DM e disfunção cognitiva em um grupo de participantes com DM2 e 48 do grupo controle, sem a enfermidade. Os instrumentos do estudo foram o WCST e o MEEM. No MEEM, não mostrou diferença significativa entre os dois grupos. No WCST os diabéticos mostraram pior desempenho. Os achados identificaram que os problemas cognitivos eram oito vezes maiores nos Diabéticos do que no grupo controle.

Foram encontrados quatro trabalhos sobre Teste Wisconsin Classificação de Cartas e DM2, a partir de 2004, porém nenhum deles abordou a temática, WCST, DM2 e idosos. O estudo que mais se aproxima é o do Watari et al. (2006) que buscou avaliar as funções executivas em adultos com DM2 e depressão maior. Os sujeitos estudados foram adultos de 30 a 80 anos de idade, com DM e depressão (idade média 57,90-DP 11,14), com DM sem depressão (idade média $=58,90 \mathrm{DP}=9,20$ ) e grupo controle (idade média 61,03 DP-14,76).
Para o diagnóstico de Depressão, foram utilizados os critérios do DSM-IV. Os resultados apontaram diferenças significativas no funcionamento cognitivo global, mostrando deficiência cognitiva maior que nos controles.

Em estudo anterior a 2004, em que foram utilizados os instrumentos Stroop Color-Word Test, Trail Making Part B, Wisconsin Card Sorting Test, and Word and Category Fluency, Delano-Wood (2002), para a avaliação da relação entre flexibilidade cognitiva depressão e ansiedade em idosos, cujos participantes foram 281 idosos, com idades entre 55 a 86 anos, os resultados apontaram que depressão e a idade predizem significativamente a inflexibilidade cognitiva.

$\mathrm{Na}$ avaliação dos padrões de déficits cognitivos em idosos com DM, Qiu et al. (2006) apresentam um estudo em 290 sujeitos, sendo 40\% destes com DM. Os resultados mostraram que as funções executivas dos idosos com DM estavam mais prejudicadas do que naqueles sem DM. Na avaliação da memória, os idosos com DM também mostraram declínio, sugerindo deficiências frontais orgânicas, que podem estar associadas à doença microvascular, que a DM pode fazer. Um estudo realizado em idosos com mais de 70 anos com DM, utilizando MMSE e Escala de Depressão Geriátrica, concluiu que idosos com DM apresentam risco de desenvolver problemas cognitivos, e, em relação ao grupo controle, os diabéticos mostraram disfunção executiva (Munshi et al., 2006).

É importante destacar que as publicações em que são utilizados os descritores Teste Wisconsin de Classificação de Cartas-WCST, idosos, DM2 são muito reduzidas. A partir dos resultados desta revisão, foram encontrados na literatura apenas três estudos que atendiam aos descritores associados. A maioria dos estudos foi descartada por estes não estarem relacionados com o objetivo aqui proposto; porém, as tabelas que ilustram este estudo mostram, mesmo através de outros instrumentos, até mesmo de neuroimagem, que a DM2 causa prejuízos significativos nas funções cognitivas.

Nesses últimos cinco anos, foram identificados cinco estudos brasileiros sobre o teste Wisconsin de classificação de cartas. A maioria utilizou de- 
lineamento transversal. A partir do ano de 2002, estudos de caso controle foram predominantemente desenvolvidos utilizando o teste Wisconsin de Classificação de Cartas em idosos com DM2.

Quanto aos estudos encontrados na literatura internacional envolvendo DM2, todos demonstram estar esta associada à disfunção cognitiva em algum grau, mesmo que não tenham sido encontrados estudos com a mesma população aqui estudada, e com o instrumento WCST. Entretanto, foram utilizados outros instrumentos de avaliação que identificaram prejuízos nas funções cognitivas associadas com a DM2 em populações de idades diferentes. Dentre os prejuízos mais evidenciados nos estudos, (Tiehuis et al., 2008) mencionam o funcionamento cognitivo prejudicado, lesões e atrofia cortical (Brands, Biessels, Haan, Kapplle \& Kessels, 2007), hipocampo prejudicado e memória declarativa (Bruehl et al., 2007).

Outros estudos identificados (Harten, Osterman, Loon, Scheltens \& Weisntein, 2007), envolvendo idosos com idade média de 73 anos, mostram que a DM é um fator de risco para diminuição da função cognitiva. Aumento de atrofia, redução do hipocampo, déficits cerebrais, de velocidade de processamento, memória, inteligência, atenção, além de reduzida velocidade psicomotora e velocidade de processamento verbal foram identificados quando comparados a grupos controles (Gold et al., 2007; Verdelho et al., 2007).

Finalizando, um estudo, utilizando o MEEM e GDS (Munshi et al., 2006) em idosos de 70 a 93 anos com DM2, encontrou déficits nas áreas de eficiência psicomotora, memória semântica, episódica e de trabalho, assim como anormalidades nas funções executivas relacionadas a comportamentos complexos, como a resolução de problemas, planejamento, organização, perspicácia raciocínio e atenção.

\section{Considerações finais}

A partir desta busca, no que tange ao objetivo proposto neste trabalho, que é verificar as características das publicações sobre o desempenho cognitivo de idosos com DM2 através do Teste Wisconsin de
Classificação de Cartas-WCST, os estudos apontaram para um prejuízo na performance em idosos com DM2.

$\mathrm{Na}$ análise da temática pesquisada, observou-se que a maioria dos abstracts identificados não apresenta clareza quanto à metodologia empregada; muitos não descrevem os instrumentos utilizados, idade da amostra; enfim, a metodologia não está explicitada; o que limitou o passo inicial de uma revisão.

No que se relaciona aos estudos empíricos encontrados, mesmo utilizando outros instrumentos, mostrou-se comprometimento em muitas funções. Isso indica que, mesmo sem a utilização do WCST, os achados destes outros instrumentos apontam para algum tipo de funcionamento cognitivo prejudicado em estudos envolvendo a DM2.

Nos estudos revisados, a maioria mostrou um grau de comprometimento relacionado com as funções cognitivas, ou seja: flexibilidade do pensamento, atenção, memória de trabalho, sugerindo, inclusive, que a DM2 acelera o processo de deterioro, aumentando a possibilidade de desenvolver demência. Também se denotou que a prevalência de depressão é mais elevada em idosos com DM2; assim, também, muitas outras complicações físicas foram identificadas.

\section{Referências}

Abel, C. G., Stein, G., Pereyra, S., Nano, G., Arakaki, T., Garretto, N. et al. (2006). Comparison study of executive functions in Parkinson's disease and degenerative cerebelar disease's patients. Ar. Neuropsiquiatr., 64 (3B), 814-826.

Akisaki, T., Sakurai, T., Takata T., Umegaki, H., Araki, A., Mizumo, S. et al. (2006). Cognitive dysfunction associates with white matter hyper intensities and subcortical atrophy on magnetic resonance imaging of the elderly diabetes mellitus Japanese elderly diabetes intervention trial (J-EDIT). Diabetes Metabolism Research an Rewiews, 22, 376-384.

Allen K. V., Frier, B. M., Strachan, M. W. (2004) .The relationship between type 2 diabetes and cognitive dysfunction: Longitudinal studies and their 
methodological limitations. Euro J. Pharmacology, 490 (1-3), 169-175.

Amarilho, C. B. \& Carlos, S. A. (2005). Executiveenterpriser, his retirement and the removal from works process. UnATI, 8 (1), 61-88.

Andrade, V. M., Santos, F. H. \& Bueno, O. F. A. (2004). Neuropsicologia hoje. São Paulo: Artes Médicas.

Argimon, I. I. L. \& Stein, L. M. (2005). Habilidades cognitivas em indivíduos muito idosos: um estudo longitudinal. Cadernos de Saúde Pública, 21 (1), 64-72.

Arvanitakis, Z., Wilson, R. S., Bienas, J. L., Evans, A. D., Li, Y. \& Bennett, D. A. (2004). Diabetes Mellitus and Risk of Alzheimer Disease and Decline in Cognitive Function. Arch. Neurol, 61, 661-666.

Arvanitakis, Z., Wilson, R. S., Li, Y., Aggarwal, N. T., Bennett, D. A. (2006). Diabetes and function in different cognitive systems in older individuals without dementia. Diabetes Care, 29 (3), 560-565.

Asimakopoulou, K. \& Hampson, S. E. (2002). Cognitive functioning and self- management in older people with diabetes. Diabetes Spectrum, 15 (2), 116-121.

Aszalós, Z. (2007). Cerebral complications of diabetes mellitus. Orvi Hetil, 148, 2371-2376.

Awad, N., Gagnon, M. \& Messier, C. (2004). The Relationship between impaired glucose tolerance, type 2 diabetes, and cognitive function. Journal of Clinical and Experimental Neuropsychology, 26 (8), 1044-1080.

Baudic, S., Dalla Barba, G., Thibaudet, M. C., Smagglhe, A., Remy, P. \& Traycov, L. (2006). Executive function deficits in early Alzheimer's disease and their relations with episodic memory. Archives of Clinical Neuropsychology, 21, 15-21.

Bauduceau, B., Bourdel-Marchasson, I., Brocker, P. \& Taillia, H. (2005). The brain of the elderly diabetic patient. Diabetes Metab., 31, 5S92-5S97.

Biessels, G. J., Kerssen, A., Haan, E. H. F. \& Kappelle, L. J. (2007). Cognitive dysfunction and diabetes: Implications for primary care. Primary Care Diabetes, 1, 187-193.

Biessels, G. J., Deary, I. J. \& Ryan, C. M. (2008). Cognition and diabetes: A lifespan perspective. Lancet Neurol, 7, 184-190.
Biessels, G. J., Haan, E. H. F., Kamal, A. \& Bleys, R. L. A. W. (2002). Ageing and diabetes: Implications for brain function. European Journal of Pharmaco$\log y, 441,1-14$.

Brands, A. M. A., Biessels, G. J., de Haan, E. H. F., Kappelle, L. J. \& Kessels, R. P. C. (2005). The effects of type 1 diabetes on cognitive performance: $\mathrm{A}$ meta-analysis. Diabetes Care, 28, 726-735.

Brands, A. M., Biessels, G. J., Kappelle, L. J., de Haan, E. H., de Valk, H. W., Algra, A. et al. (2007). Cognitive functioning and brain MRI in patients with type 1 and type 2 diabetes mellitus: A comparative study. Dementia and Geriatric Cognitive Disorder, 23, 343-350.

Bruce, D. G., Harrington, N., Davis, W. A. \& Davis, T. M. E. (2001). Dementia and its associations in type 2 diabetes mellitus: The Fremantle Diabetes Study. Diabetes Research and Clinical Practice, 53, 165-172.

Bruehl, H., Rueger, M., Dziobek, I., Sweat, V., Tirsi, A., Javier, E. et al. (2007). Hypothalamic-PituitaryAdrenal Axis dysregulation and memory impairments in Type 2 Diabetes. The Journal of Clinical Endocrinology \& Metabolism, 92 (7), 2439-2445.

Costa, M. F. L. \& Veras, R. (2003). Saúde e envelhecimento. Cad. Saúde Pública, 19 (3), 793-798.

Cosway, R., Strachan, M. W. J., Dougall, A., Frier, B. M. \& Deary, I. J. (2001). Cognitive function and information processing in Type 2 diabetes. Diabetes Uk. Diabetic Medicine, 18, 803-810.

Coutinho, E. S. F. (2003). Meta-análise. Em R. A. Medronho, D. M. Carvalho, V. V. Bloch, R. R. Luiz \& G. L. Werneck (Orgs.), Epidemiologia (pp. 447-55). São Paulo: Atheneu.

Cunha, P. J., Nicastri, S., Gomes, L. P., Moino, R. M. \& Pesluso, M. A. (2004). Alterações neuropsicológicas em dependentes de cocaína/crack internados: dados preliminares. Rev. Bras. Psiquiatr., 26 (2), 103-106.

Cunha, J. A., Trentini, C. M., Argimon, I. L., Oliveira, M. S., Werlang, B. G., Prieb, R. G. (2005). Teste Wisconsin de Classificação de Cartas - Adaptação e Padronização Brasileira. São Paulo: Casa do Psicólogo.

Delano-Wood, M. L. (2002). The relationship between cognitive flexibility, depression, and anxiety 
in older adults. Michigan State University, 41(2), 621-691.

Fauber, J. (2006). Dementia and Diabetes: Poorly controlled insulin may contribute to. Milwaukee Journal Sentinel, set, 11.

Ferreira, S. R. G., Almeida, B., Siqueira, A. F. A. \& Khawali, C. (2005). Intervenções na prevenção do Diabetes Mellitus Tipo 2: É viável um programa populacional em Nosso Meio?Arq Bras. Endocrinol. Metab., 49 (4), 479-484.

Foss-Freitas, M. C. \& Foss, M. C. (2003). Cetoacidose diabética e estado hiperglicêmico hiperosmolar. Medicina, Ribeirão Preto, 36, 389-393.

Freitas, E., Py, L., Cançado, F. A. X. \& Gorzoni, M. (2006). Tratado de Geriatria e Gerontologia. Rio de Janeiro: Koogan.

Gold, S. M., Dziobek, I., Sweat, V., Tirsi, A., Rogers, K., Bruehl, H. et al. (2007) Hippocampal damage and memory impairments as possible early brain complications of type 2 diabetes. Diabetologia, 50, 711-719.

Grodstein, F., Chen, J., Wilson, R. S. \& Manson, J. E. (2001). Type 2 Diabetes and Cognitive Function in Community-Dwelling Elderly Women. Diabetes Care, 24 (6), 1060-1065.

Hamdan, A. C. \& Bueno, O. F. A. (2005). Relações entre controle executivo e memória episódica verbal no comprometimento cognitivo leve e na demência tipo Alzheimer. Estudos de Psicologia (Natal), 10 (1), 63-71.

Harten, B., Osterman, J. M., Loon, B. J., Scheltens, P. \& Weinstein, H. C. (2007) Brain Lesions on MRI in Elderly Patients with Type 2 Diabetes Mellitus. European Neurology, 57, 70-74.

Harten, B., Oosterman, J., Muslimovic, D., Van Loon, B. J. , Scheltens, P. \& Weinstein, H. C. (2007). Cognitive impairment and MRI correlates in the elderly patients with type 2 diabetes mellitus. Age Ageing, 36 (2), 164-170.

Hassing, L. B., Hofer, S. M., Nilsson, S. E., Berg, S., Pedersen, N. L., McClearn, G. et al. (2004). Comorbid typem 2 diabetes mellitus and hypertension exacerbates cognitive decline; evidence from a longitudinal study. Age and Ageing - British Geriatrics Society, 33 (4), 355-361.
Hassing, L. B., Grant, M. D., Hofer, S. M., Pedersen, N. L., Nilsson, S. E., Berg, S. et al. (2004). Type 2 diabetes mellitus contributes to cognitive decline in old age: A longitudinal population-based study. Journal of the International Neuropsychological Society, 10, 599-607.

Huber, S. J. (1992). Magnetic resonance imaging correlates of executive functions impairment in multiple sclerosis. Neuropsychiatry, Neuropsychology and Behavioral Neurology, 5 (1), 33-36.

Kanaya, A. M., Barrett-Connor, E., Gildengorin, G. \& Yaffe, K. (2004). Change in cognitive function by glucose tolerance status in older adults. Arch. Intern. Med., 164 (12), 1327-1333.

Keller, M. \& Werlang, B. S. G. (2005). Flexibilidade na resolução de problemas em tentadores de suicídio. J. Bras. Psiquiaty., 54 (2), 128-136.

Kodl, C. T. \& Seaquist, E. R. (2008). Cognitive Dysfunction and Diabetes Mellitus. Endocrine Reviews, 29 (4), 494-511.

Kouta, Y., Sakurai, T. \& Yokono, K. (2006). Cognitive dysfunction and dementia associated with elderly diabetes. Nippon Rinsho, 64 (1), 119-123.

Kuo, H. K., Jones, R. N., Milberg, W. P., Tennstedt, S., Talbot, L., Morris, J. N. et al. (2005). Effect of blood pressure and diabetes mellitus on cognitive and physical functions in older adults: A longitudinal analysis of the advanced cognitive training for independent and vital elderly cohort. Journal of the American Geriatrics Society, 53 (7), 1154-1161.

Lerário, A. C., Coretti, F. M. L. M., Oliveira, S. F., Betti, R. T. B., Bastos, M. S. C. B., Ferri, L. A. F. et al. (2008). Avaliação da prevalência do diabetes e da hiperglicemia de estresse no infarto agudo do miocárdio. Arq. Bras. Endrocrinol. Metab., 52 (3), 465-472.

Lezak, M. D. (2005). Neuropsychological assessment (4 ed.). New York: Oxford University Press.

Lezak, M. D. (1995). Neuropsychological assessment (3 ed.). New York: Oxford University Press.

Maineri,N.L., Xavier,F.M.F., Berleze,M.C.C., Moriguchi,E.H. (2007).Fatores de Risco para Doença Cerebrovascular e Função Cognitiva em Idosos. Arq.Bras. Cardiol., 89(3),158-162. 
Malloy-Diniz, L. F., Sedo, M., Fuentes, D. \& Leite, W. B. (2006). Neuropsicologia: teoria e prática. Porto Alegre: Artes Médicas.

Manschot, S.M., Brands, A.M.A, Grond, J., Kessels,R.P.C., Algra,A., Kappelle, L.J., et al.(2006). Brain Magnetic Ressonance imaging Correlates of Impaired Cognition in Patients With Type 2 Diabetes. Diabetes, vol. 55, april.

McGuire, L.C., Ford, E.S., Ajami, U. (2006). The impact of cognitive functioning on mortality and the development of functional disability in older adults with diabetes:the second longitudinal study on aging. BMC Geriatrics, 6, 8. doi: 10.1186/14712318-6-8.

Messier, C. (2005). Impact of impaired glucose tolerance and type 2 diabetes on cognitive aging. Neurobiology of Aging, 26S, S26-S30.

Mogi, N., Umegaki, H., Hattori, A., Maeda, N., Miura, H., Kuzuya, M. et al. (2003). Cognitive function in Japanese elderly with type 2 diabetes mellitus. Journal of Diabetes and Its Complications, 18 (1), 42-46.

Munshi, M., Grande, L., Hayes, M. \& Ayres, D. (2006). Cognitive dysfunction is associated with poor diabetes control in older adults. Diabetes Care, 29 (8), 1794-1799.

Nascimento, R.F.L. (2007). Depressão e o desempenho cognitivo em idosos através do teste Wisconsin classificação de cartas (WCST). [documento impresso e eletrônico]. Diss. Mestrado em Psicologia, Fac. de Psicologia, PUCRS, Porto Alegre. Dispon. http://tede.pucrs.br/tde_busca/arquivo. php? codArquivo $=961$

Qiu, W. Q., Price, L. L., Hibberd, P., Buell, J., Collins, L., Leins, D. et al. (2006). Executive dysfunction in homebound older people with diabetes mellitus. Journal of the American Geriatrics Society, 54, 496-501.

Rigoni, M. S., Oliveira, M. S., Moraes, J. F. D. \& Zambom, L. F. (2007). O consumo de maconha na adolescência e as conseqüências nas funções cognitivas. Psicologia em estudo, 12 (2), 267-275.

Rodriguez-Gomez, J. R., Davila-Martinez, M. G., \& Collazo-Rodriguez, L. C. (2006). Factor structure of the Beck Depression Inventory-Second Edition
(BDI-II) with Puerto Rican elderly. P R Health Sci J., 25 (2), 127-132.

Rocca, C. C. A. \& Lafer, B. (2006). Neuropsicologia: teoria e prática. Porto Alegre: Artes Médicas.

Ryan, C.M., Freed, M.I., Rood, J.A., Cobitz,A.R., Waterhouse,B.R., Strachan,M.W.J. (2006) Improving Metabolic Control Leads to Better Working Memory in Adults With Type 2 Diabetes. Diabetes Care, vol.29,n 2, February.

Sakurai, T. \& Yokono, K. (2006). Comprehensive studies of cognitive impairment of the elderly with type 2 diabetes. Geriatr. Gerontol. Int, 6, 159-164.

Sastre, A. A. \& Evans, J. G. (2008). Effect of the treatment of Type II diabetes mellitus on the development of cognitive impairment and dementia. Cochrane Database of Systematic Reviews, 1.

Seyfaddini, R. (2006). Ti: Cognitive function in Diabetes Mellitus Patients. American Journal of Apllied Sciences, 3 (1), 1682-1684.

Sinclair, A. J., Girlingb, A. J., Bayerc, A. J. (2000). Cognitive dysfunction in older subjects with diabetes mellitus: Impact on diabetes self-management and use of care services. Diabetes Research and Clinical Practice, 50, 203-212.

Starr, V. L. \& Convit, A. (2007). Diabetes, sugar-coated but harmful to the brain. Current Opinion in Phamacology, 5, 638-642.

Strauss, E., Sherman, E. M. S. \& Spreen, O. (2006). A compendium of neuropsychological tests (3 ed.). New York: Oxford U. Press.

Suzuki, M., Umegaki, H., Uno, T., Oyen, O., Mogi, N., Maeno, H. et al. (2006). Association between insulin resistance and cognitive function in elderly diabetic patients. Geriatry Gerontology, 6, 254-259.

Tiehuis, A. M., Vincken, K. L., Van Den Berg, E., Hendrikse, J., Manschot, S. M., Mali, W. P. T. M. et al. (2008). Diabetologia, 51, 1321-1326.

Tiarapú-Ustrárroz , J. \& Muñoz-Céspedes, J. M. (2005). Memória y funciones ejecutiva. Rev. Neuro., 41 (8), 475-484.

Van den Berg, E., Kessels, R. P. C., Kappelle, L. J., de Haan, E. H. F. $\mathcal{E}$ Biessels, G. J. (2006). Drugs of Today, 42 (11), 741-754.

Verdelho, A., Madureira, S., Ferro, J. M., Basile, A. M., Chabriat, C. H., Erkinjuntti, T. et al. (2007). Di- 
fferential impact of cerebral white matter changes, diabetes, hypertension and stroke on cognitive performance among non disabled elderly. The LADIS study. J. Neurol. Neurosurg. Psychiatry, 78, 1325-1330.

Watari, K., Letamendi, A., Thompson-Elderkin, V., Haroon, E., Miller, J., Darvin, D. et al. (2006). Cognitive function in adults with type 2 diabetes and major depression. Arquives of Clinical Neuropsychology, 21, 787-796.
Wechsler, D. (2004). Wais-III: Escala de Inteligência Wechsler para Adultos: Manua/ David Wechsler; Adaptação e Padronização de uma amostra Brasileira ( $1^{\underline{a}}$ ed); Elizabeth do Nascimento. São Paulo: Casa do Psicólogo.

Wild, S., Roglic, G., Green, A., Sicree, R. \& King, H. (2004) Global Prevelence of Diabetes. Diabetes Care, 27 (5), 1047-1053.

Zhao, Y. \& Liu, F. (2000). A preliminary investigation of cognitive dysfunction in type II diabetes patients. Chinese Mental Health Journal, 14 (1), 21-24. 
